\title{
Effectiveness of preoperative decolonization with nasal povidone iodine in Chinese patients undergoing elective orthopedic surgery: a prospective cross- sectional study
}

\author{
H-M. Peng, L-C. Wang, J-L. Zhai, X-S. Weng, B. Feng and W. Wang \\ Department of Orthopedic Surgery, Peking Union Medical College Hospital, Chinese Academy of Medical Sciences and \\ Peking Union Medical College, Beijing, China
}

\begin{abstract}
Staphylococcus aureus colonization in the nares of patients undergoing elective orthopedic surgery increases the potential risk of surgical site infections. Methicillin-resistant $S$. aureus (MRSA) has gained recognition as a pathogen that is no longer only just a hospital-acquired pathogen. Patients positive for MRSA are associated with higher rates of morbidity and mortality following infection. MRSA is commonly found in the nares, and methicillin-sensitive S. aureus (MSSA) is even more prevalent. Recently, studies have determined that screening for this pathogen prior to surgery and diminishing staphylococcal infections at the surgical site will dramatically reduce surgical site infections. A nasal mupirocin treatment is shown to significantly reduce the colonization of the pathogen. However, this treatment is expensive and is currently not available in China. Thus, in this study, we first sought to determine the prevalence of MSSA/MSRA in patients undergoing elective orthopedic surgery in northern China, and then, we treated the positive patients with a nasal povidone-iodine swab. Here, we demonstrate a successful reduction in the colonization of $S$. aureus. We propose that this treatment could serve as a cost-effective means of eradicating this pathogen in patients undergoing elective orthopedic surgery, which might reduce the rate of surgical site infections.
\end{abstract}

Key words: Nasal methicillin-resistant; Staphylococcus aureus; Orthopedic surgery; Nasal povidone iodine; Prospective crosssectional study

\section{Introduction}

The rate of Staphylococcus aureus colonization in the nares of the general population is reported to be as high as $30 \%$ (1). In addition, genotyping studies reveal that as high as $80 \%$ of $S$. aureus infections are caused by the patient's own nasal flora (2). Furthermore, there is an association between the presence of nasal $S$. aureus and an increased risk of surgical site infection (SSI), which has been extensively demonstrated in orthopedic surgery (3-5).

Over the last 2 decades, methicillin-resistant $S$. aureus (MRSA) has gained recognition as a community-acquired pathogen and is no longer only a hospital-acquired pathogen. Importantly, MRSA is associated with higher rates of morbidity and mortality following infection (6).

The increased morbidity associated with SSI translates directly into increased costs associated with medical care. It is reported that surgical site infection is associated with a 2-week increase in hospital stay, double the rate of hospitalization, and triple the overall cost of treatment on average (3).
Thus, screening and decolonization of $S$. aureus carriers prior to surgery has emerged as an important method for diminishing staphylococcal infections at the surgical site.

Surveillance studies (7-10) suggest that there is a high variation in the prevalence of MRSA infections depending on its location. Specifically, 0.6 to $6 \%$ of the general population has MRSA nasal colonization compared to methicillinsensitive $S$. aureus (MSSA) nasal carriers, which make up 20 to $36.4 \%$ of the population. A previous review examining patients undergoing elective orthopedic surgery (11) concluded that screening initiatives needed to be intensified at all levels in order to contain the spread of this pathogen. Furthermore, it was emphasized that the screening initiatives at the regional and hospital levels would be the most effective.

An intranasal application of mupirocin is proven to be effective for the decolonization of this microbe and the prevention of invasive $S$. aureus infections in patients

Correspondence: X-S.Weng: <xshweng@medmail.com.cn>

Received May 17, 2017 | Accepted September 18, 2017 
receiving long-term dialysis treatment (11-13). However, mupirocin is not currently available in China. In addition, due to its cost and the concern about patient compliance and the development of resistance, recent reports suggest that a preoperative nasal application of a povidone-iodine solution may be more efficacious than nasal mupirocin in preventing SSIs (14). The efficiency of the application of a nasal povidone-iodine swab for the decolonization of $S$. aureus is yet unknown in China.

Moreover, to the best of our knowledge, data regarding the prevalence and distribution of MSSA and MRSA in patients undergoing elective orthopedic surgery in China are lacking, and Chinese hospitals have no established guidelines for screening and decolonizing patients for the presence of MSSA and/or MRSA pathogens. Therefore, the main aim of this study was to assess the prevalence of MSSA/MRSA in the patients admitted to our institute. Furthermore, our secondary aim was to determine whether the current treatment protocols result in the successful decolonization of MSSA/MRSA.

\section{Patients and Methods}

\section{Patients}

This was a prospective cross-sectional study conducted in the Department of Orthopedics at Peking Union Medical College Hospital between August 2015 and February 2016. The eligible procedures included joint arthroplasty and spine fusion procedures requiring at least 3 days of overnight in the hospital. A consecutive series of patients undergoing elective orthopedic surgery during the abovementioned interval participated in the present study. This study was approved by the Institutional Ethics Committee of Peking Union Medical College Hospital, and informed consent was obtained from the patients before the swabbing.

The minimum sample size needed was calculated to be 457 patients in order to detect the prevalence with a $2 \%$ precision, and this was based on the notion that the MRSA prevalence rate was expected to be $5 \%$, as indicated in previous studies (11). The study subjects were patients who were admitted to the orthopedics ward within $24 \mathrm{~h}$ of admission to the hospital.

In addition, a standardized questionnaire was completed for each patient by a personal interview in order to evaluate the patients' characteristics and, therefore, assess possible risk factors and possible sources of transmission. The patient characteristics included gender, from urban or rural area, date of admission, reason for hospitalization, chronic underlying disease, presence of pets at home, smoking history, number of previous hospitalizations as well as the number of hospitalizations in the 3 months prior to this admission, antibiotic use in the 3 months prior to this admission (including the dosage, name and duration of use, if known) and hospitalization of a family member in the preceding 3 months (Table 1 ).
Table 1. Baseline characteristics of the screened patients.

\begin{tabular}{lc}
\hline Characteristic & Screened patients $(\mathrm{n}=545)$ \\
\hline Mean age (years) & $41.8 \pm 13.7$ \\
Male (\%) & $280(51 \%)$ \\
ASA score at admission & $473 / 545$ \\
$1-2$ & $72 / 545$ \\
3 & \\
Underlying disorder & $134 / 545$ \\
Hypertension & $142 / 545$ \\
History of smoking & $70 / 545$ \\
Alcohol & $112 / 545$ \\
Diabetes mellitus type 1 or 2 & $22 / 545$ \\
Renal insufficiency & $34 / 545$ \\
Liver-function disorder & $12 / 545$ \\
Malignant condition & $31 / 545$ \\
Skin disease & \\
Procedure & $307 / 545$ \\
Joint arthroplasty & $230 / 545$ \\
Spine fusion & $8 / 545$ \\
Other & \\
\hline
\end{tabular}

ASA: American Society of Anesthesiologists.

\section{Exclusion criteria}

Patients with any of the following were excluded from the study: hospitalization within past 3 months; a history of antibiotic use within the past 3 months; being in close proximity to other ill patients in the hospital or in their household; allergy to povidone iodine and an infectious indication for surgery.

\section{Screening}

The patients were screened for nasal MRSA/MSSA colonization within $24 \mathrm{~h}$ of admission. The specimens were collected from both of the anterior nares by one cotton swab that was moistened with sterile saline. The walls of the vestibules of the anterior nares were thoroughly swabbed for 10-15 s. The swabs were then inoculated on both chromID MRSA agar (MRSA; BioMérieux S.A, France) and blood agar. The colonies suspected as MRSA were confirmed by MALDI-TOF MS (BioMérieux), and methicillin susceptibility was determined using cefoxitindisk test. All of the patients in the study were then reswabbed on the day of surgery using the same technique described above. This was done to determine whether the decolonization protocol (described below) eradicated MSSA/MRSA colonization.

\section{Decolonization}

The patients who were positive for MSSA and/or MRSA underwent the decolonization procedure. For this, 5\% povidone-iodine nasal swabs were used in both nostrils twice a day for 5 days prior to the surgery. The nurses prepped the patient's nostrils for approximately $30 \mathrm{~s}$ each 
using separate applicators. This process was then repeated using 2 additional applicators for a total application time of $1 \mathrm{~min}$ per naris ( $2 \mathrm{~min}$ total). The patients were also instructed to take chlorhexidine gluconate baths for the 5 days before the surgery. The patients who were negative for MRSA and MSSA colonization did not receive any decolonization treatment.

\section{Prophylaxis}

The primary antimicrobial prophylaxis was cefuroxime $(1.5 \mathrm{mg})$. The patients with a reported $\beta$-lactam allergy received clindamycin $(600 \mathrm{mg})$, and those colonized with MRSA received vancomycin (1000 $\mathrm{mg})$. Antibiotic infusion was started within $1 \mathrm{~h}$ of the incision ( $2 \mathrm{~h}$ for vancomycin) and was re-dosed according to the hospital guidelines. The patients were monitored for hospital-acquired $S$. aureus infection by means of microbiologic cultures. The physicians were encouraged to obtain culture samples if infection was suspected.

\section{Statistical analysis}

All the statistical analyses were performed using the software SPSS (version 19.0; SPSS, Inc., USA). The statistical analyses comparing the pre-operative results to the results from the day of surgery were performed using the McNemar test. Statistical significance was defined as $\mathrm{P}<0.05$.

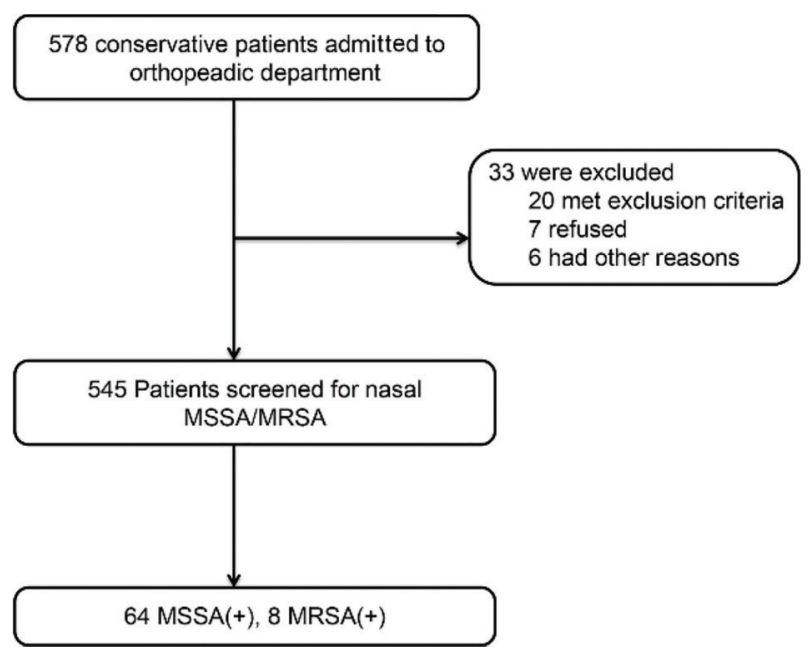

Figure 1. Study enrollment. MRSA: methicillin-resistant $S$. aureus; MSSA: methicillin-sensitive $S$. aureus.

\section{Results}

\section{Patient screening}

During the study period, 545 patients underwent screening for both MRSA and MSSA by routine cultures. A total of 578 elective surgical procedures were performed during the same period, yielding a successful screening rate of $94.2 \%$. Among the screened patients, 64 patients $(11.74 \%)$ were identified as MSSA carriers, and 8 patients $(1.28 \%)$ were identified as MRSA carriers. Thus, a total of 72 patients were treated with $5 \%$ povidone-iodine nasal swabs for decolonization (Figure 1).

\section{Decolonization results}

The decolonization treatment was given for 5 days before the surgery. On the day of surgery, all patients (including the patients that were previously negative for MSSA and MRSA) were screened again to determine whether the MSSA or MSRA was decolonized from the patients' nostrils. The result from the day of the surgery screening showed that none of the patients were positive for MRSA colonization, showing a $100 \%$ successful decolonization. However, 11 patients were positive for MSSA colonization, which was $2 \%$ of the total patients (545) and was approximately a reduction of $92.6 \%$ compared to the screening before the decolonization, which was $11.74 \%$ of the total patients. Among the 11 patients that were positive for MSSA at the day of surgery screening, 7 were negative for MSSA before the decolonization treatment, and 4 had persistently positive MSSA colonization cultures. It should be noted that one of the patients who was persistently colonized for MSSA refused the decolonization protocol. Thus, there were only 3 patients who underwent the decolonization treatment and were persistently positive for MSSA at the time of surgery compared to 64 that were positive before the treatment. The eradication of the MSSA colonization was $94 \%$, while the eradication of the MRSA colonization was $100 \%$ (Table 2). The compliance rate for the patients undergoing the decolonization protocol was $98.4 \%$ (only 1 patient refused).

\section{Cost analysis}

The average cost per patient for the MRSA screening was US\$11 (SD, 0), and the average cost per patient for the hand-made povidone-iodine nasal swabs was US\$1 (SD, 0; Table 3).

Table 2. Comparison of screening results before and after decolonization.

\begin{tabular}{lccc}
\hline & Pre-decolonization $(n=545)$ & Post-decolonization $(n=545)$ & $P$ \\
\hline MSSA cases & $64(11.7 \%)$ & $3(0.6 \%)$ & $<0.001$ \\
MRSA cases & $8(1.3 \%)$ & $0(0)$ & $<0.000$ \\
\hline
\end{tabular}

MRSA: methicillin-resistant $S$. aureus; MSSA: methicillin-sensitive $S$. aureus. 
Table 3. Comparison of the cost (in US dollars) of different procedures.

\begin{tabular}{lrc}
\hline & Screening & Decolonization \\
\hline Culture + mupirocin & $\$ 27.12$ & $\$ 130$ \\
PCR + mupirocin & $\$ 121.16$ & $\$ 130$ \\
Our procedures & $\$ 10.00$ & $\$ 1$ \\
\hline
\end{tabular}

\section{Discussion}

In this study, we assessed the prevalence of MSSA/ MRSA in patients undergoing elective orthopedic surgery and treated the positive patients with $5 \%$ povidone-iodine nasal swabs to decolonize these pathogens from the anterior nares. The patients were screened within $24 \mathrm{~h}$ of their admission to the orthopedic department at our institute in northern China to determine the success of the decolonization. We chose to swab the anterior nares as they are the natural niches of $S$. aureus and are the most consistent areas from which the organism has been isolated (15). Other sites, such as the throat, axilla, groin and/or rectum, are alternative sites for colonization. However, these sites were excluded due to the concern about patient participation.

Two methods commonly used for the identification of $S$. aureus include culturing and polymerase chain reaction (PCR). Culturing the bacteria on chromogenic agar is a less expensive test, while PCR is considered the 'gold standard' for MRSA detection (15-17). Our decision to use the culture method was based on the economic standpoint (less expensive for the cost of material and labor) and the desired speed to provide the test result (1-2 days).

In our study, the prevalence of MRSA colonization was $1.28 \%$, and the overall rate of $S$. aureus in the nasal region was $13.2 \%$. Both of these rates were lower than the nasal carriage rates reported in other studies from China or abroad $(1,9,18-22)$. MRSA incidence in elective orthopedic surgery was also lower than reported incidence in other surgeries $(23,24)$. Costantini et al. $(23)$ estimated a prevalence of $4.2 \%$ in children undergoing heart surgery. Another study by Ramirez et al. (24) reported a high rate of $6.4 \%$ in patients who underwent major gastrointestinal operations. Some previous large, population-based surveillance studies showed that colonization varies between different groups (ethnicity, gender, and age), with higher rates in whites, in men $(7,9)$ and elderly patients $(20)$. Our patients did not have a history of a recent hospital admission or other risk factors associated with MRSA colonization. The nasal swabs were collected within $24 \mathrm{~h}$ of admission to exclude colonization of the nares resulting from hospitalization. The screening population was different from other studies. Thus, the preoperative orthopedic outpatient colonization rates with MSSA and MRSA in our study population seem to reflect the real rates of the general population in Northern China.

The results of our study also demonstrated that utilizing a simple and less expensive decolonization protocol significantly reduced the colonization of MSSA/MRSA in nasal carriers. Only 3 of the patients who were positive for MSSA pre-operatively and underwent decolonization were persistently positive on the day of surgery. One patient refused to receive the decolonization protocol, and thus, the positive result on the day of surgery was expected. Another finding from the present study was that 7 of the patients who were positive for MSSA on the day of surgery were not positive during the pre-operative screen. The reason for this may be due to the fact that the preoperative swabs were not sensitive enough to detect $S$. aureus colonization, and this might be improved by utilizing the PCR method instead of routine cultures $(11,22)$ or by swabbing from multiple sites (15).

The application of nasal mupirocin to decolonize $S$. aureus and prevent subsequent SSIs is proven effective in many control studies $(11,13,20,25,26)$. Chen AF et al. found that the decolonization protocols using intranasal mupirocin and chlorhexidine washes are effective for reducing MRSA/MSSA colonization (13). Decolonization of $S$. aureus using intranasal mupirocin and chlorhexidine in patients undergoing elective orthopedic surgery could significantly increase the risk of postoperative SSI (11,25). Agarwala et al. (20) reported that mupirocin was an effective treatment in clearing MRSA from the nares in adult patients undergoing orthopedic surgery in urban India, thereby reducing the incidence of MRSA SSI. In a double-blind, randomized, placebo-controlled study, use of mupirocin nasal ointment for perioperative eradication of $S$. aureus nasal carriage was significantly more effective, and the rate of endogenous $S$. aureus infections was 5 times lower than in the placebo group (26). However, nasal mupirocin is currently not available in China, and its compliance may be problematic due to its side effects and additional cost $(14,27)$. A previous study suggested that preoperative hand-made nasal povidone iodine with topical chlorhexidine demonstrated a similar effectiveness, compared to nasal mupirocin, to decolonize $S$. aureus (13). The application of mupirocin to decolonize the nares of patients prior to orthopedic surgery was demonstrated as a cost-effective intervention. Currently, the nasal mupirocin available for application to the nasal mucosa cost approximately US $\$ 130$ /course (14), while our hand-made nasal povidone iodine cost approximately US\$1/application, given the equal decolonization efficacy. Our method provides more value, as it is more suitable for developing countries, for universal surveillance and for the decolonization of patients undergoing elective orthopedic surgery.

Our study has several limitations. First, there is potential underestimation of the $S$. aureus carrier rate because we only focused on the anterior nares and did not include other sites, such as the throat, axilla, groin, and/or rectum. 
However, previous studies documenting a linkage between colonization and infection have cited nasal colonization, rather than reservoirs from these other sites, as the risk factor for infection. Second, our study design was cross sectional, which makes predictions of changes in colonization with time impossible. Third, this study only evaluated the effectiveness of the treatment using hand-made nasal povidone iodine swabs and a chlorhexidine body wash, but did not evaluate other treatment modalities, including oral antibiotics. Finally, this study detected the presence of MRSA/MSSA by a culture swab, rather than by PCR, which could increase the sensitivity of detection.

In conclusion, this study, for the first time, identified the prevalence of MRSA/MSSA in patients undergoing elective orthopedic surgery in Northern China. After identifying the positive patients, they were treated with a hand-made

\section{References}

1. Kuehnert MJ, Kruszon-Moran D, Hill HA, McQuillan G, McAllister SK, Fosheim G, et al. Prevalence of Staphylococcus aureus nasal colonization in the United States, 2001-2002. $J$ Infect Dis 2006; 193: 172-179, doi: 10.1086/499632.

2. Wertheim HF, Vos MC, Ott $A$, van Belkum $A$, Voss $A$, Kluytmans JA, et al. Risk and outcome of nosocomial Staphylococcus aureus bacteraemia in nasal carriers versus non-carriers. Lancet 2004; 364: 703-705, doi: 10.1016/ S0140-6736(04)16897-9.

3. Whitehouse JD, Friedman ND, Kirkland KB, Richardson WJ, Sexton DJ. The impact of surgical-site infections following orthopedic surgery at a community hospital and a university hospital: adverse quality of life, excess length of stay, and extra cost. Infect Control Hosp Epidemiol 2002; 23: 183-189, doi: 10.1086/502033.

4. Yano K, Minoda $Y$, Sakawa A, Kuwano $Y$, Kondo K, Fukushima $W$ et al. Positive nasal culture of methicillinresistant Staphylococcus aureus (MRSA) is a risk factor for surgical site infection in orthopedics. Acta Orthop 2009; 80: 486-490, doi: 10.3109/17453670903110675.

5. Berthelot P, Grattard F, Cazorla C, Passot JP, Fayard JP, Meley R, et al. Is nasal carriage of Staphylococcus aureus the main acquisition pathway for surgical-site infection in orthopaedic surgery? Eur J Clin Microbiol Infect Dis 2010; 29: 373-382, doi: 10.1007/s10096-009-0867-5.

6. Melzer M, Eykyn SJ, Gransden WR. Chinn S. Is methicillinresistant Staphylococcus aureus more virulent than methicillin-susceptible S. aureus? A comparative cohort study of British patients with nosocomial infection and bacteremia. Clin Infect Dis 2003; 37: 1453-1460, doi: 10.1086/379321.

7. Tiemersma EW, Bronzwaer SL, Lyytikainen O, Degener JE, Schrijnemakers $P$, Bruinsma $N$, et al. Methicillin-resistant Staphylococcus aureus in Europe, 1999-2002. Emerg Infect Dis 2004; 10: 1627-1634, doi: 10.3201/eid1009.040069.

8. Price CS, Williams A, Philips G, Dayton M, Smith W, Morgan S. Staphylococcus aureus nasal colonization in preoperative orthopaedic outpatients. Clin Orthop Relat Res 2008; 466: 2842-2847, doi: 10.1007/s11999-008-0337-x.

9. Gorwitz RJ, Kruszon-Moran D, McAllister SK, McQuillan G, McDougal LK, Fosheim GE, et al. Changes in the prevalence nasal povidone iodine swab, which successfully eradicated the detection of MSRA and significantly reduced the colonization of MSSA. This treatment was as effective as nasal mupirocin. However, mupirocin is not available in China at this time and it is significantly more expensive. We propose that a hand-made nasal povidone iodine swab should be evaluated in larger cohorts of orthopedic surgery patients to determine its efficacy in eradicating MRSA/MSSA colonization, which could significantly reduce $\mathrm{SSI}$.

\section{Acknowledgements}

This study was supported by National High-tech R\&D Program (863 Program) with grant No. 2015AA020316 and No. 2015AA033601 from People's Republic of China.

of nasal colonization with Staphylococcus aureus in the United States, 2001-2004. J Infect Dis 2008; 197: 1226-1234, doi: $10.1086 / 533494$

10. Mertz D, Frei R, Jaussi B, Tietz A, Stebler C, Fluckiger U, et al. Throat swabs are necessary to reliably detect carriers of Staphylococcus aureus. Clin Infect Dis 2007; 45: 475-477, doi: 10.1086/520016.

11. Kim DH, Spencer M, Davidson SM, Li L, Shaw JD, Gulczynski D, et al. Institutional prescreening for detection and eradication of methicillin-resistant Staphylococcus aureus in patients undergoing elective orthopaedic surgery. J Bone Joint Surg Am 2010; 92: 1820-1826, doi: 10.2106/JBJS.I. 01050.

12. Rao N, Cannella BA, Crossett LS, Yates AJ Jr, McGough $\mathrm{RL}$, et al. Preoperative screening/decolonization for Staphylococcus aureus to prevent orthopedic surgical site infection: prospective cohort study with 2-year follow-up. J Arthroplasty 2011; 26: 1501-1507, doi: 10.1016/j.arth.2011.03.014.

13. Chen AF, Heyl AE, Xu PZ, Rao N, Klatt BA. Preoperative decolonization effective at reducing staphylococcal colonization in total joint arthroplasty patients. J Arthroplasty 2013; 28: 18-20, doi: 10.1016/j.arth.2013.03.036

14. Phillips M, Rosenberg A, Shopsin B, Cuff G, Skeete F, Foti $A$, et al. Preventing surgical site infections: a randomized, open-label trial of nasal mupirocin ointment and nasal povidone-iodine solution. Infect Control Hosp Epidemiol 2014; 35: 826-832, doi: 10.1086/676872.

15. Weiser MC, Moucha CS. The current state of screening and decolonization for the prevention of Staphylococcus aureus surgical site infection after total hip and knee arthroplasty. J Bone Joint Surg Am 2015; 97: 1449-1458, doi: 10.2106/ JBJS.N.01114.

16. Shenoy ES, Lee H, Cotter JA, Ware W, Kelbaugh D, Weil $E$, et al. Impact of rapid screening for discontinuation of methicillin-resistant Staphylococcus aureus contact precautions. Am J Infect Control 2016; 44: 215-221, doi: 10.1016/ j.ajic.2015.08.019

17. Hogan B, Rakotozandrindrainy R, Al-Emran H, Dekker D, Hahn A, Jaeger A, et al. Prevalence of nasal colonisation by methicillin-sensitive and methicillin-resistant Staphylococcus 
aureus among healthcare workers and students in Madagascar. BMC Infect Dis 2016; 16: 420, doi: 10.1186/s12879016-1733-6.

18. Zhang J, Gu FF, Zhao SY, Xiao SZ, Wang YC, Guo XK, et al. Prevalence and molecular epidemiology of Staphylococcus aureus among residents of seven nursing homes in Shanghai. PLoS One 2015; 10: e0137593, doi: 10.1371/journal.pone. 0137593.

19. Chen B, Dai X, He B, Pan K, Li H, Liu X, et al. Differences in Staphylococcus aureus nasal carriage and molecular characteristics among community residents and healthcare workers at Sun Yat-Sen University, Guangzhou, Southern China. BMC Infect Dis 2015; 15: 303, doi: 10.1186/s12879015-1032-7.

20. Agarwala S, Lad D, Agashe V, Sobti A. Prevalence of MRSA colonization in an adult urban Indian population undergoing orthopaedic surgery. J Clin Orthop Trauma 2016; 7: 12-16, doi: 10.1016/j.jcot.2015.08.004.

21. Askarian M, Zeinalzadeh A, Japoni A, Alborzi A, Memish ZA. Prevalence of nasal carriage of methicillin-resistant Staphylococcus aureus and its antibiotic susceptibility pattern in healthcare workers at Namazi Hospital, Shiraz, Iran. Int $J$ Infect Dis 2009; 13: e241-e247, doi: 10.1016/j.jijid.2008.11.026.

22. Durmaz G, Sanci O, Oz Y, Guven K, Kiremitci A, Aksit F. Methicillin-resistant $S$. aureus colonization in intensive care unit patients: Early identification and molecular typing. $J$ Infect Dev Ctries 2016; 10: 465-471, doi: 10.3855/jidc.6575.

23. Costantini ST, Lach D, Goldfarb J, Stewart RD, Foster CB. Staphylococcus aureus colonization in children undergoing heart surgery. World J Pediatr Congenit Heart Surg 2013; 4: 267-70, doi: 10.1177/2150135113480530.

24. Ramirez MC, Marchessault M, Govednik-Horny C, Jupiter D, Papaconstantinou HT. The impact of MRSA colonization on surgical site infection following major gastrointestinal surgery. J Gastrointest Surg 2013; 17: 144-152; discussion p.152, doi: 10.1007/s11605-012-1995-2.

25. Akhtar A, Kadir H, Chandran P. Surgical site infection risk following pre-operative MRSA detection in elective orthopaedic surgery. J Orthop 2014; 11: 117-120, doi: 10.1016/ j.jor.2014.07.001.

26. Kalmeijer MD, Coertjens $H$, van Nieuwland-Bollen PM, BogaersHofman D, de Baere GA, Stuurman A, et al. Surgical site infections in orthopedic surgery: the effect of mupirocin nasal ointment in a double-blind, randomized, placebo-controlled study. Clin Infect Dis 2002; 35: 353-358, doi: 10.1086/341025.

27. Torres EG, Lindmair-Snell JM, Langan JW and Burnikel BG. Is preoperative nasal povidone-iodine as efficient and costeffective as standard methicillin-resistant Staphylococcus aureus screening protocol in total joint arthroplasty? J Arthroplasty 2016; 31: 215-218, doi: 10.1016/j.arth.2015.09.030. 